Rabaska

Revue d'ethnologie de l'Amérique française

\title{
Institut du patrimoine (Université du Québec à Montréal)
}

\section{Lisa Baillargeon et Yves Bergeron}

Volume 18, 2020

URI : https://id.erudit.org/iderudit/1072961ar

DOI : https://doi.org/10.7202/1072961ar

Aller au sommaire du numéro

Éditeur(s)

Société québécoise d'ethnologie

ISSN

1703-7433 (imprimé)

1916-7350 (numérique)

Découvrir la revue

Citer ce document

Baillargeon, L. \& Bergeron, Y. (2020). Institut du patrimoine (Université du Québec à Montréal). Rabaska, 18, 442-444. https://doi.org/10.7202/1072961ar d'utilisation que vous pouvez consulter en ligne. 
carrière, il a diffusé le fruit de ses recherches en publiant des ouvrages destinés au grand public, en réalisant des expositions ou en participant à des séries documentaires diffusées à la télévision. Connu du grand public et reconnu par ses pairs, Michel Lessard a joué un rôle déterminant dans le domaine du patrimoine. Centrée sur les thèmes qui traversent la carrière de Michel Lessard, cette journée d'étude a permis de rappeler que la question du patrimoine est au cœur de l'histoire de l'UQÀm depuis sa création et de souligner la contribution de cette jeune université à la culture québécoise. Le comité scientifique responsable de ce colloque était composé de Lisa Baillargeon, Anne Bénichou, Yves Bergeron, René Bouchard et Jean-Pierre Pichette.

\section{Conclusion}

Malgré la pandémie qui a mis un frein à certaines de ses activités, la SQE entend poursuivre sa mission avec une vigueur renouvelée au cours de l'année 2020-2021 grâce, entre autres, à l'engagement de ses membres et à la collaboration de ses partenaires et commanditaires.

SuZANNE MARCHAND

\section{Institut du patrimoine}

Université du Québec à Montréal

Pavillon R, 3760

Téléphone : (514) 987-3000, poste 5139

C.P. 8888, Succ. Centre-ville, Montréal (Qc) Courriel : baillargeon.lisa@uqam.ca H3C $3 \mathrm{P} 8$

Toile : institutpatrimoine.uqam.ca

\section{Sous le signe de la commémoration}

Dans le cadre du 50 anniversaire de l'UQÀM, l'Institut du patrimoine (IP) a amorcé à l'automne 2019 une série d'entretiens avec des personnalités qui ont marqué le patrimoine de l'institution dans différents secteurs du patrimoine : Paul-André Linteau et le patrimoine montréalais ; Raymond Montpetit et la création des programmes de muséologie ; France Vanlaethem et la défense du patrimoine bâti contemporain ; Esther Trépanier et le patrimoine artistique ; ainsi que Marie Beaulieu et le patrimoine de la danse. Chacun de ces entretiens a été mené par de jeunes chercheurs et diffusé en direct sur la page Facebook de l'Institut. Ces entretiens se poursuivront au cours de la prochaine année.

En collaboration avec la Société québécoise d'ethnologie, le département d'histoire de l'art et les cycles supérieurs en muséologie, l'Institut a tenu le 17 février 2020 une journée d'étude consacrée à la carrière et l'œuvre de Michel Lessard qui a marqué le monde du patrimoine sur plusieurs décennies. Le témoignage d'anciens collègues et de jeunes chercheurs ont confirmé l'impact de Michel Lessard sur le patrimoine bâti, le patrimoine mobilier, l'histoire de la photographie, la muséologie et le cinéma ethnographique.

L'Institut a maintenu son programme de colloques et de conférences. Soulignons notamment le Séminaire international sur la gouvernance des musées (Sénégal- 
Québec) qui s'est tenu en deux temps : du 11 au 14 juin à Dakar et du 4 au 7 novembre à l'UQÀM ; il était organisé conjointement avec plusieurs partenaires dont la Chaire de recherche sur la gouvernance des musées et le droit de la culture, le Musée de la femme - Longueuil, le Musée de la femme - Dakar, le Musée Théodore-Monod d'art africain, le CÉLAT, la Faculté de sciences politiques et de droit de l'UQÀM, le Conseil international des musées, le Musée des beaux-arts de Montréal, le Musée de Pointeà-Callière et l'Écomusée du fier monde. L'IP a présenté une série de conférences, dont Alfred H. Barr Jr et la naissance de la collection du Moma à New York par Jérôme Glicenstein (Université Paris 8) ; Médiation culturelle et muséologie : approche collaborative aux Forges de Montréal par Catherine Charron ; Les enjeux d'une nouvelle définition des musées pour le Conseil international des musées par Alberto Garlandini (Iсом-Italie) ; et Parcours d'une « Promenade en Enfer » : les livres censurés comme objets d'études ethnologiques par Pierrette Lafond.

\section{Veille en patrimoine}

L'Institut poursuit sa veille en patrimoine, enrichie notamment par des sites de centres et de groupes de recherche (CÉLAT, CRILCQ, Laboratoire d'histoire et de patrimoine de Montréal - UQÀM, etc.), d'associations (AMC, SMQ, CQPV, ICOFOM, ICOM, SQE, etc.) et de lieux patrimoniaux. L'équipe est restée également à l'affût de ce qui est publié sur les réseaux sociaux (Facebook, Instagram, Linkedin, etc.) : des pages et des groupes de professionnels ou d'initiatives citoyennes ainsi que des pages officielles de musées, de lieux patrimoniaux, de groupes de recherche et d'associations sont régulièrement explorées.

Pour l'année 2019-2020, l'Institut a maintenu l'édition de ses infolettres, de septembre à mai. La crise sanitaire a eu un effet sur le travail de veille. L'actualité en patrimoine ainsi que les initiatives sur la Toile se sont révélées foisonnantes et l'Institut, dans les circonstances, souhaitait témoigner de ces initiatives et des changements subis par cette crise. La page Facebook de 1'Institut permet de suivre l'actualité quotidienne en patrimoine par deux publications. Les publications francophones, les événements de l'Institut, celles concernant des membres ainsi que les publications scientifiques restent les plus populaires. Nous avons observé cette année une augmentation notable des interactions des abonnés avec la page, notamment avec plus de commentaires et de partages des publications. Les vidéos diffusées en direct favorisent la visibilité générale de l'Institut. Notre première rencontre en ligne sur le documentaire Hubert Reeves : conteurs d'étoiles a rejoint un large public.

La crise sanitaire actuelle a accéléré le traitement et la diffusion des archives audiovisuelles des différentes activités de l'Institut (colloques, entretiens, rencontres, conférences, etc.), hébergées sur la chaîne YouTube : sous l’onglet « Archives », du site « Institut Patrimoine de l'UQÀM », l'équipe ajoutera graduellement les captations de ces activités ; on y retrouve la rencontre avec John Porter, ancien directeur du Musée national des beaux-arts du Québec.

\section{Le Réseau patrimoines de l'Université du Québec}

Lancé officiellement en 2018, le RÉPUQ regroupe des chercheurs en patrimoine des 
différentes composantes de l'Université du Québec. Le Réseau avait proposé un colloque pour l'édition 2020 de l'ACFAS à Sherbrooke ayant pour titre « 20 ans après le rapport Arpin : les nouveaux territoires du patrimoine » afin de souligner le $20^{\mathrm{e}}$ anniversaire du Rapport Arpin (2000-2020) sur le patrimoine culturel du Québec. Cette rencontre scientifique proposait de se pencher sur l'évolution du concept de patrimoine. Comme le congrès de l'ACFAs a été annulé, les membres du Réseau ont choisi de reporter le congrès en mai 2021 à l'Université de Sherbrooke. [Voir le rapport 2019 et le texte sur ce réseau]

\section{Ciné-rencontres}

Le cycle des ciné-rencontres de l'année a été coordonné par Alexis Lemieux (doctorant en muséologie, médiation, patrimoine, membre de l'Institut et du LABDOC). Au programme, deux ciné-rencontres tenues à l'UQÀM (films : La Bataille de Forillon, 4 décembre 2019 ; et «Louis Pelletier ». Hommage au film de Maurice Proulx, 5 mars 2020) ; et deux autres en diffusion virtuelle pendant les premiers mois de la pandémie de la Covid-19 au Québec : Hubert Reeves : conteur d'étoile (2002), 22 avril 2020 ; Un musée dans la ville (2011), Luc Bourdon, 20 mai 2020). Depuis mars 2020, 1'Ip propose de façon hebdomadaire à ses membres des films à visionner, en lien avec le patrimoine.

\section{Projet 2020-2021}

L'Institut a poursuivi le travail collaboratif avec les cinq autres instituts de l'UQÀm afin de proposer un cadre de gestion des instituts. Le Conseil des instituts a planifié un projet collectif afin de développer une synergie, d'amorcer une pratique de travail interinstituts et d'aborder collectivement des sujets de grande ampleur, complexes dans une perspective multidisciplinaire. Le thème retenu pour l'année 2020-2021 - Habiter le monde ou s'abriter du monde? - est inspiré de la crise du Coronavirus. Enfin, l'Institut est partenaire avec l'UQÀM, l'Université de Montréal, l'Université Laval, l'Université du Québec en Outaouais, l'Université du Québec à TroisRivières et d'autres universités pour l'organisation du $43^{\mathrm{e}}$ symposium international d'ICOFOM, qui se tiendra du 15 au 19 mars 2021 à Montréal, Québec et GatineauOttawa, sur le thème La Décolonisation de la muséologie : musées, métissages et mythes d'origine.

Au cours de la prochaine année, l'Ip entend mettre l'accent sur la création d'une concentration de premier cycle en patrimoine qui regrouperait des cours existants dans plusieurs programmes, dont histoire de l'art, histoire, études autochtones, études urbaines, design, géographie, théâtre, cinéma, danse et gestion.

Après avoir accepté la direction de l'Institut en 2017 avec l'objectif de lui donner une nouvelle orientation, Yves Bergeron a terminé son mandat le 30 mai 2020. Lisa Baillargeon, de l'École des sciences de la gestion, qui était la présidente du conseil scientifique, assure la direction de l'Institut depuis le $1^{\text {er }}$ juin.

Lisa Baillargeon et Yves Bergeron 